\title{
PERCORSI SCOLASTICI E SCELTE FORMATIVE DEI GIOVANI LATINOS IN LOMBARDIA
}

\author{
CHIARA CAVAGNINI
}

Università Cattolica del Sacro Cuore. Milano (Italia)

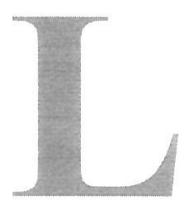

\section{RESUMEN}

a escuela es uno de los principales agentes de socialización. En el caso de los hijos de latinoamericanos migrantes residentes en la provincia de la Lombardía, se hace necesario un análisis de los datos disponibles y un acercamiento a la realidad educativa, a las características de los estudiantes y a los principales problemas. En este artículo se presenta un análisis descriptivo de la situación y se apuntan algunas líneas de trabajo futuro.

Palabras clave: escolarización de hijos de migrantes, Italia, escuela, socialización.

\section{INTRODUZIONE}

Negli ultimi anni le scuole italiane di ogni ordine e grado hanno visto aumentare in modo rilevante fra i propri iscritti il numero degli alunni con cittadinanza non italiana, tanto che, nell'anno scolastico 2003/2004 gli alunni stranieri hanno raggiunto le 282.683 unità, il doppio rispetto a quelle registrate nel 2000/2001, con un'incidenza sul totale della popolazione scolastica del 3,49\%. Le presenze appiano diversificate rispetto ai vari ordini di scuola ${ }^{1}$ : se nella primaria è accolto il $40,78 \%$ del totale degli alunni stranieri, nella secondaria di primo grado si registra il $23,89 \%$, seguita dalla scuola dell'infanzia, nella quale i numeri sono in continuo aumento, con il $19,44 \%$ e infine dalla secondaria di secondo grado con il 15,89\%. L'incidenza sul totale degli

1 La recente riforma dell'ordinamento scolastico italiano, denominata riforma Moratti (n. 53 del 2003), prevede la suddivisione degli ordini di scuola in: scuola dell'infanzia (3-5 anni, la cui frequenza non è obbligatoria), scuola primaria (6-10), scuola secondaria di primo grado (11-13), scuola secondaria di secondo grado (dai 14), ampliando a 12 anni il diritto-dovere all'istruzione e alla formazione. 
alunni nei vari ordini di scuola si attesta su valori superiori alla media sia per la primaria (con il 4,47\%), sia per la secondaria di primo grado, con il 4,01\%, sia per la percentuale, leggermente inferiore, della scuola dell'infanzia, che è del $3,83 \%$, mentre è decisamente al di sotto della media quella delle secondarie di secondo grado $(1,87 \%)$. Sia i numeri assoluti che l'incidenza variano da città a città, mettendo in evidenza una situazione "a macchia di leopardo" che vede fra i primi quattro posti per numerosità delle presenze tre città del nord Italia: i numeri più consistenti si registrano infatti a Milano, seguita da Roma, Torino e Brescia.

Tale presenza pone la scuola italiana di fronte a nuove sfide che riguardano la conoscenza, non solo a livello quantitativo, di questi alunni e degli immigrati di seconda generazione, le modalità di intendere la loro integrazione, nonché una riflessione intorno alle strategie didattiche e ai contenuti dei curricoli, sinora impostati secondo un'ottica eurocentrica.

L'istituzione scolastica italiana si colloca infatti all'inizio di un percorso già intrapreso dai paesi europei da maggior tempo aree di destinazione dei flussi migratori, percorso che, se non progettato e valutato, rischia di mettere in campo soluzioni apparentemente innovative, a cui tuttavia sottostà un'ottica assimilativa, che richiede agli alunni stranieri di integrarsi conformandosi alle richieste e alle aspettative del contesto scolastico.

Il nodo, infatti, che in questi anni la scuola si trova a sciogliere ruota intorno alle modalità con cui affrontare due diverse istanze: da un lato la garanzia della fruizione di diritti fondamentali e la costruzione di forme di appartenenza a partire da una base egualitaria; dall'altro l'assicurazione di percorsi di crescita che tengano conto delle diverse individualità dei soggetti: a fronte di un'uguaglianza di opportunità deve essere pertanto garantita l'attenzione alla diversità dei bisogni e delle inclinazioni ${ }^{2}$. Cruciale è quindi il modo di intendere l'integrazione sociale e scolastica, che spesso, in un'ottica di tipo assimilazionista, va nella direzione di inglobare e ridurre la differenza e non, piuttosto, di mettere tutti gli alunni nelle condizioni di poter fruire appieno delle risorse, di disporre delle chances per la piena realizzazione del sé.

L'analisi dei contesti scolastici multiculturali, pertanto, se da un lato può essere finalizzata alla creazione di interventi mirati, rivolti agli alunni stranieri, dall'altro apre la questione del ripensamento

2 Besozzi E., Insegnare in una società multietnica: tra accoglienza, indifferenza e rifuto, in Giovannini G. (a cura di), Allievi in classe stranieri in città. Una ricerca sugli insegnanti di scuola elementare di fronte all'immigrazione, F. Angeli, Milano 1996. 
dell'impostazione generale del sistema scolastico, delle pratiche educative sedimentate al suo interno; si evidenzia quindi l'importanza non solo di riconoscere le specificità culturali dei gruppi di minoranza, ma di attuare una riflessione intorno alla cultura che oggi esprime l'istituzione scolastica.

Il presente lavoro si pone come un approfondimento nell' ambito dei risultati emersi dall'indagine promossa dall'Osservatorio Regionale per l'integrazione e la multietnicità riguardante la presenza degli alunni con cittadinanza non italiana nelle scuole statali e non statali della Lombardia nell'anno scolastico $2003 / 2004^{3}$, regione italiana che registra il più alto numero di studenti stranieri e che da sola comprende circa un quarto delle presenze dell'intero territorio nazionale. In particolare, prendendo in esame i dati relativi alla presenza dei giovani Latinos nelle scuole lombarde, si sono volute analizzare le specificità di questa parte della popolazione scolastica straniera, sia in base alle differenze territoriali registrate nelle undici città della regione, sia in base alla loro distribuzione nei vari ordini di scuola. Data la numerosità, fra i Latinos di seconda generazione, di alunni peruviani ed ecuadoriani, cittadinanza quest'ultima di recente immigrazione nel territorio lombardo e che ha registrato negli ultimi anni un notevole aumento delle presenze, si è focalizzata l'attenzione sui loro percorsi scolastici e sui processi legati alla scelta dell'indirizzo di studi superiori, particolarmente significativa nell'ottica del futuro inserimento lavorativo e sociale.

\section{LA PRESENZA DEI LATINOS NELLE SCUOLE STATALI E NON STATALI DELLA LOMBARDIA}

Dall'indagine Insieme a scuola $3^{4}$ risulta che nelle scuole statali e non statali lombarde sono presenti 71.114 alunni con cittadinanza straniera, corrispondenti al $6,7 \%$ dell' intera popolazione scolastica; tale incidenza è quasi il doppio di quella registrata a livello nazionale. Le presenze variano naturalmente da città a città, in base alle caratteristiche che fanno di ogni polo urbano e del suo territorio un ambiente consono all'insediamento di nuclei familiari; è interessante notare come negli ultimi anni le aree metropolitane, come Milano, pur avendo il primato per numero assoluto di presenze di alunni stranieri, abbiano subito una flessione, in particolare rispetto all'incidenza sul totale della popolazione scolastica, mentre, in parallelo, le città di provincia hanno

3 L'anno scolastico italiano inizia a settembre e termina a giugno dell'anno successivo.

4 L'indagine è stata effettuata nelle scuole statali $e$ non statali di ogni ordine e grado della Lombardia presenti negli elenchi del Ministero dell'Istruzione, Universitả e Ricerca, le quali hanno compilato un questionario, direttamente disponibile sul sito del Ministero, per ogni alunno straniero iscritto. 
visto aumentare in numero considerevole gli alunni con cittadinanza non italiana nelle proprie scuole.

Guardando alla distribuzione nei vari ordini di scuola, le presenze maggiori si registrano nella scuola primaria, in cui è concentrato il $41,4 \%$ degli alunni, seguiti dalla scuola dell'infanzia $(23,2 \%)$, dalla secondaria di primo grado, con il $22,8 \%$ e dalla secondaria di secondo grado, che accoglie il minor numero di alunni stranieri, con il $12,6 \%$ (Tab. 1).

Tab.1. Alunni con cittadinanza non italiana nelle scuole della Lombardia per ordine di scuola. Anno scol. 2003/2004.

\begin{tabular}{|l|c|c|}
\hline Ordine di scuola & V.a. & $\%$ \\
\hline Dell'Infanzia & 16.494 & 23,2 \\
Primaria & 29.423 & 41,4 \\
Secondaria di I grado & 16.202 & 22,8 \\
Secondaria di II grado & 8.995 & 12,6 \\
\hline Totale & 71.114 & 100,0 \\
\hline
\end{tabular}

Fonte: Besozzi E., Tiana M. T. (a cura di), Insieme a scuola 3, Fondazione Ismu, Milano 2005.

L'incidenza sul totale della popolazione scolastica è maggiore nelle scuole dell'infanzia e diminuisce progressivamente negli ordini scolastici successivi, a fronte di un numero crescente di minori nati sul territorio italiano ${ }^{5}$ da genitori stranieri, dato che, da un lato, è legato alla crescita della presenza femminile, in particolare di alcune aree geografiche, fra gli immigrati presenti in Lombardia e dall'aumento dei ricongiungimenti familiari e, dall'altro, testimonierebbe la sempre maggiore stabilizzazione di nuclei familiari stranieri sul territorio ${ }^{6}$.

La distribuzione per continente di provenienza vede al primo posto in Lombardia gli alunni provenienti dall'Europa non comunitaria (20.610 presenze), seguiti a brevissima distanza da coloro che provengono dall'Africa, con 20.533 alunni; vi sono poi gli alunni asiatici, pari a 15.052 unità, coloro che provengono dal continente americano, con 12.011 presenze, gli europei comunitari (1.706) e, infine, gli alunni provenienti dall'Oceania o senza cittadinanza (42 unità). Tale andamento riflette in modo sostanziale quello italiano generale, con alcune differenze per quanto riguarda le singole aree di provenienza: a livello nazionale si afferma infatti in modo più netto il primato degli alunni dell'Europa non comunitaria mentre, in percentuale, è minore la presenza di asiatici e americani (Tab. 2).

5 Secondo la legislazione italiana, in base allo ius sanguinis, chi nasce in Italia da genitori stranieri è straniero e può richiedere la cittadinanza al compimento del $18^{\circ}$ anno.

6 Cfr. Mazzi D., Presentazione dei risultati generali, in Besozzi E., Tiana M. T. (a cura di), Insieme a scuola 3. La terza indagine regionale, Fondazione Ismu, Milano 2005. 
Tab. 2. Alunni con cittadinanza non italiana nelle scuole italiane e della Lombardia per continente di provenienza. Anno scol. 2003/2004

\begin{tabular}{l|r|r|r|r}
\hline \multirow{2}{*}{ CONTINENTE DI PROVENIENZA } & \multicolumn{2}{|c|}{ ITALIA } & \multicolumn{2}{c|}{ LOMBARDIA } \\
\cline { 2 - 5 } Unione Europea & V.A. & \multicolumn{1}{c|}{$\%$} & \multicolumn{1}{c|}{.A. } & \multicolumn{1}{c}{$\%$} \\
Europa non UE & 7.419 & 2,6 & 1.706 & 2,4 \\
Africa & 123.685 & 43,8 & 20.610 & 29,5 \\
Asia & 73.103 & 25,9 & 20.533 & 29,4 \\
America & 41.904 & 14,8 & 15.052 & 21,5 \\
Oceania e apolidi & 36.164 & 12,8 & 12.011 & 17,2 \\
TOTALE & 408 & 0,1 & 42 & 0,0 \\
\hline
\end{tabular}

Fonte: Besozzi E., Tiana M. T. (a cura di), Insieme a scuola 3, Fondazione Ismu, Milano 2005.

In particolare per quanto riguarda le provenienze dall'America, è importante sottolineare come dei 12.011 alunni presenti in Lombardia solo 173 provengano dall'America settentrionale (Canada e U.S.A.), mentre ben 11.803, pari al 98,3\%, dall'America centro-meridionale. In Lombardia, regione nelle cui scuole sono accolte 165 nazionalità, con una grande varietà di lingue e codici culturali con cui le istituzioni scolastiche devono confrontarsi, sono rappresentate tutte le cittadinanze latino-americane, seppur con dei numeri assoluti molto diversi e distribuite in modo differenziato nelle diverse province della regione. Fra le prime dieci (Tab. 3), che da sole raggiungono il 96,6\% del totale degli alunni dell'America centro-meridionale iscritti nelle scuole lombarde, il primato spetta agli alunni provenienti dall'Ecuador, nazionalità le cui presenze hanno avuto negli ultimi anni una forte crescita, con 4.223 unità, pari al 35,8\% dei Latinos, seguiti a distanza dagli alunni di origine peruviana $(1.495$, corrispondenti al $26 \%$ ). Fra le prime due nazionalità e le altre cittadinanze dei Latinos presenti in Lombardia si nota un certo divario: al terzo posto si collocano i brasiliani, con una percentuale dell' $8,7 \%$, e, a seguire, i minori provenienti dall'Argentina (5,4\%), El Salvador (5\%), Bolivia (4,7\%), Colombia (4,6\%), Repubblica Dominicana $(3,2 \%)$, Cuba $(1,9 \%)$ e Cile $(1,3 \%)$.

Guardando alla distribuzione degli alunni delle prime dieci nazionalità dell'America centro-meridionale rispetto al genere, è interessante notare come per la quasi totalità di queste cittadinanze la percentuale di presenza femminile è superiore alla media regionale, che si attesta intorno al $47,4 \%$, ad eccezione solamente degli alunni provenienti da E1 Salvador, fra i quali le femmine sono comunque il $47,3 \%$ del totale. Per cinque nazionalità inoltre, rispettivamente Repubblica Dominicana, Ecuador, Cuba, Cile e Colombia, la percentuale delle femmine supera quella dei maschi, toccando il 54,1\% per le alunne di origine colombiana.

La ripartizione dei Latinos nei vari ordini di scuola rispecchia l'andamento regionale solamente per quanto concerne la maggioranza 
delle presenze, collocate nella scuola primaria; il dato significativo riguarda invece la scuola secondaria di secondo grado, che supera la scuola dell'infanzia, in particolare per alcune nazionalità, quali il Perù'; tale dato potrebbe essere interpretato sia alla luce del luogo di nascita dei minori presenti in Lombardia (se in Italia o all'estero), sia agli anni di permanenza in Italia, che favorirebbero il proseguo degli studi, sia alla modalità di concepire l'istruzione da parte delle famiglie provenienti da alcune aree rispetto alla scuola dell'infanzia, dato il carattere di non obbligatorietà di tale ordine di scuola, che accoglie minori fino ai cinque anni d'età.

Tab. 3 Alunni provenienti dall'America-centro meridionale nelle scuole della Lombardia per principali paesi di cittadinanza e ordine di scuola. Anno scol. 2003/2004

\begin{tabular}{|c|c|c|c|c|c|c|c|}
\hline Paesi di cittadinanza & $\mid$ Dell'Infanzia $\mid$ & Primaria & $\begin{array}{l}\text { Secondaria } \\
\text { di I grado }\end{array}$ & $\begin{array}{l}\text { Secondaria } \\
\text { di II grado }\end{array}$ & Totale & \begin{tabular}{|c|} 
di cui: \\
femmine
\end{tabular} & $\begin{array}{c}\% \text { su totale } \\
\text { continente/area }\end{array}$ \\
\hline \multicolumn{7}{|l|}{ America centro-meridionale } & 98,3 \\
\hline Ecuador & 678 & 1.780 & 1.162 & 603 & 4.223 & 2.140 & 35,8 \\
\hline Peru' & 593 & 967 & 743 & 767 & 3.070 & 1.495 & 26,0 \\
\hline Brasile & 137 & 463 & 235 & 188 & 1.023 & 502 & 8,7 \\
\hline Argentina & 130 & 273 & 138 & 92 & 633 & 301 & 5,4 \\
\hline El Salvador & 124 & 230 & 139 & 100 & 593 & 281 & 5,0 \\
\hline Bolivia & 118 & 203 & 125 & 106 & 552 & 272 & 4,7 \\
\hline Colombia & 81 & 225 & 131 & 110 & 547 & 273 & 4,6 \\
\hline Rep. Dominicana & 42 & 135 & 131 & 74 & 382 & 207 & 3,2 \\
\hline Cuba & 13 & 104 & 78 & 26 & 221 & 111 & 1,9 \\
\hline Cile & 23 & 66 & 40 & 24 & 153 & 78 & 1,3 \\
\hline Altri America centro-meridionale & 71 & 165 & 95 & 75 & 406 & 212 & 3,4 \\
\hline Totale America centro-meridionale & 2.010 & 4.611 & 3.017 & 2.165 & 11.803 & 5.872 & 100,0 \\
\hline
\end{tabular}

Fonte: Besozzi E., Tiana M. T. (a cura di), Insieme a scuola 3, Fondazione Ismu, Milano 2005.

Per quanto riguarda le diverse città della regione, la presenza dei Latinos, come precedentemente accennato, varia considerevolmente da zona a zona; tale dato è legato in particolare alla presenza di catene migratorie e all'etnicizzazione di alcuni lavori, quale ad esempio quello domestico per le donne dell'Ecuador o quello legato all'allevamento nel settore agricolo per gli indiani, maggiormente richiesti nel mercato del lavoro in alcune aree e meno in altre.

Guardando alle undici province che costituiscono la Lombardia, in tutte, tranne Brescia, fra le prime quindici cittadinanze maggiormente rappresentate sono presenti alunni provenienti dall'America centromeridionale; in particolare, se si prendono in considerazione le prime trenta cittadinanze, il maggior numero di nazionalità proveniente da queste aree è presente a Milano, provincia con il più alto numero

7 Le presenze dei peruviani nella scuola secondaria di secondo grado superano anche quelle della secondaria di primo grado. 
assoluto -con alunni provenienti da Ecuador, Perù, Brasile, El Salvador, Colombia, Argentina, Repubblica Dominicana, Cile- per un totale di 7.512 alunni $^{8}$, corrispondenti al $26,2 \%$ di tutti gli alunni con cittadinanza non italiana presenti nella provincia. L'area milanese si discosta dalle medie regionali con dieci punti in più per quanto riguarda le provenienze dall'America'; nella provincia di Milano è quindi particolarmente significativo il numero degli alunni provenienti dall'America centromeridonale, in particolare per quanto riguarda ecuadoriani e peruviani: basti pensare che essi, che si collocano rispettivamente al primo e quarto posto fra i principali paesi di cittadinanza nella provincia, da soli raggiungono le 5.900 unità, pari al $20,6 \%$ del totale degli alunni stranieri. La situazione più particolare riguarda, in specifico, gli alunni dell'Ecuador, che nel 2000 erano meno di 500 e nell'anno scolastico $2003 / 2004$ sono più che quintuplicati ${ }^{10}$. Segue poi Varese, le cui scuole accolgono sette nazionalità dell'America centro-meridionale (Ecuador, Perù, El Salvador, Brasile, Argentina, Repubblica Dominicana, Colombia) e di queste sei si collocano fra le prime quindici per numerosità, con 722 giovani Latinos che costituiscono il 15,7\% del totale degli alunni stranieri presenti in questa provincia. Con altrettante cittadinanze troviamo Pavia, con 348 alunni provenienti da Ecuador, Argentina, Perù, Repubblica Dominicana, Colombia, Brasile, Cuba, che corrispondono al $12,7 \%$ del totale, con un'incidenza minore rispetto a Varese.

Come visualizzato nei grafici sotto riportati, l'incidenza degli alunni provenienti dall'America centro-meridionale presenti nelle scuole statali della Lombardia varia considerevolmente da città a città, a fronte di numeri assoluti molto diversificati: si passa infatti dai 28.623 alunni con cittadinanza non italiana della provincia di Milano (Grafico 1) agli 11.897 della provincia di Brescia, ai numeri decisamente più contenuti di Lecco, con 2055 alunni, Lodi (1.704) e Sondrio (497 alunni) (Grafico 2).

Guardando alle singole nazionalità, i numeri più consistenti nella quasi totalità delle città riguardano ecuadoriani e peruviani, ad eccezione di Bergamo, in cui spiccano, al quarto posto per numerosità delle presenze, gli alunni provenienti dalla Bolivia.

Se guardiamo alla presenza dei Latinos fra le nazionalità maggiormente rappresentate considerando tutta la Lombardia, è possibile notare come, fra le prime dieci, che sommate danno il $66 \%$

8 Gli alunni Latinos presenti a Milano superano le 100 unità per tutte le cittadinanze censite.

9 Giovannini G., Le cittadinanze, in Besozzi E., Tiana M. T. (a cura di), op. cit., p. 69.

10 lvi, p. 72 
del totale delle cittadinanze, siano presenti Ecuador e Perù, collocati rispettivamente al quarto e al settimo posto, con un'incidenza del $6 \%$ e del $4,4 \%$ sul totale della popolazione scolastica straniera. (Tab.4).

Grafico 1. Alunni stranieri nella provincia di Milano:

Latinos e altre cittadinanze nel capoluogo di provincia. Anno scol. 2003/2004.

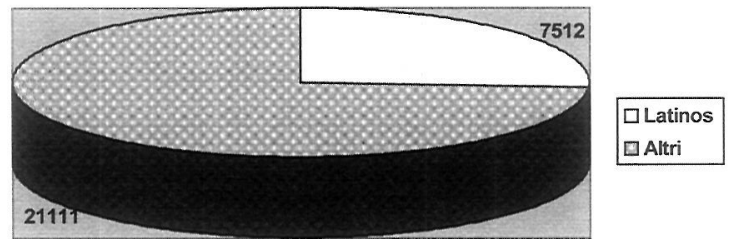

Fonte: Besozzi E., Tiana M. T. (a cura di), Insieme a scuola 3, Fondazione Ismu, Milano 2005.

Grafico 2. Alunni stranieri nelle scuole della Lombardia:

Latinos e altre cittadinanze nelle singole città (escluso il capoluogo). Anno scol. 2003/2004

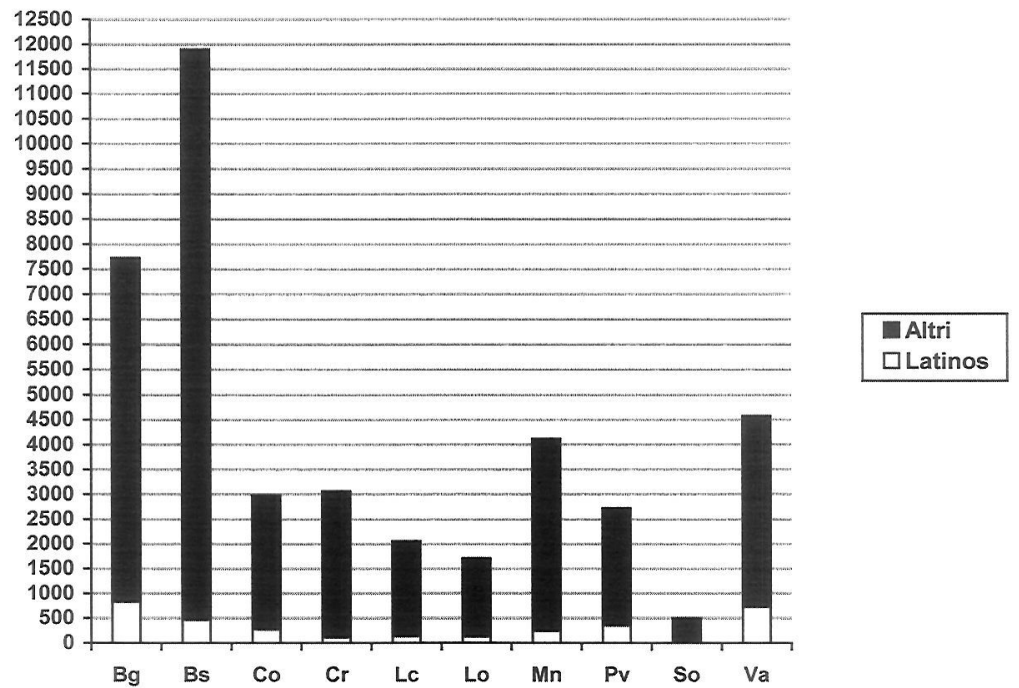

Fonte: Besozzi E., Tiana M. T. (a cura di), Insieme a scuola 3, Fondazione Ismu, Milano 2005.

$\mathrm{Da}$ qui in poi l'analisi si concentrerà soprattutto su queste due nazionalità, numericamente maggiormente rappresentate nelle scuole statali e non statali della Lombardia, in particolare per quanto riguarda i percorsi scolastici degli alunni provenienti da queste due aree e le scelte formative riguardanti la scuola secondaria di secondo grado. 
Tab. 4. Alunni con cittadinanza non italiana nelle scuole della Lombardia per principali paesi di cittadinanza. Anno scol. 2003/2004

\begin{tabular}{l|c|c} 
PAESI DI CITTADINANZA & V.A. & $\%$ \\
\hline Marocco & 10.019 & 14,3 \\
Albania & 9.453 & 13,5 \\
Romania & 4.480 & 6,4 \\
Ecuador & 4.223 & 6,0 \\
Cina & 3.948 & 5,6 \\
Filippine & 3.540 & 5,1 \\
Peru' & 3.070 & 4,4 \\
India & 2.888 & 4,1 \\
Egitto & 2.774 & 4,0 \\
Pakistan & 1.744 & 2,5 \\
Altri paesi & 23.815 & 34,0 \\
TOTALE & $\mathbf{6 9 . 9 5 4}$ & $\mathbf{1 0 0 , 0}$ \\
\hline
\end{tabular}

Fonte: Besozzi E., Tiana M. T. (a cura di), Insieme a scuola 3, Fondazione Ismu, Milano 2005.

\section{PERCORSI SCOLASTICI E SCELTE FORMATIVE DEI GIOVA- NI ECUADORIANI E PERUVIANI}

L'analisi dei percorsi e della riuscita scolastica permette di porre in luce alcuni aspetti del rapporto instauratosi fra alunni stranieri e scuola di accoglienza, nonché di cogliere le azioni positive (buone pratiche) orientate all'accoglienza e all'inserimento nell'ottica di rispondere efficacemente all' istanza che coniuga l'attenzione alle differenze con la garanzia di uguali opportunità educative per tutti gli alunni ${ }^{11}$.

A livello regionale la ricerca pone in luce un andamento positivo per quanto concerne la frequenza scolastica, regolare per quasi il 97\% dei ragazzi con cittadinanza non italiana e per più del $90 \%$ dei bambini iscritti alla scuola dell'infanzia -non obbligatoria-, dato che attesta una regolarità dei percorsi dal punto di vista della frequentazione della vita delle scuole della Lombardia ${ }^{12}$. Numerosi sono comunque gli inserimenti ad anno scolastico iniziato, legati in particolare alla mobilità delle famiglie sul territorio o all'iscrizione di ragazzi neo-arrivati e giunti direttamente dal paese d'origine ${ }^{13}$. La situazione appare invece diversificata e presenta elementi problematici più evidenti per quanto riguarda gli esiti scolastici, che danno conto di percorsi spesso irregolari, connessi ad una pluralità di fattori legati alla vicenda personale di ogni

11 Besozzi E., Percorsi ed esiti scolastici degli alunni con cittadinanza non italiana. Un'analisi dei fattori in gioco, in Ministero dell'Istruzione, dell'Università e della Ricerca, Indagine sugli esiti degli Alunni con Cittadinanza Non Italiana, gennaio 2005.

12 Tiana M. T., I percorsi e la riuscita scolastica, in Besozzi E., Tiana M. T. (a cura di), op. cit., p. 74.

13 Secondo la normativa italiana la scuola è tenuta ad accogliere in ogni momento dell'anno scolastico i minori che ne facciano richiesta, anche quelli in situazione di irregolarità. 
allievo. È infatti importante sottolineare come la riuscita scolastica non debba essere letta solamente alla luce dei dati statistici, ma, piuttosto, vada considerata, sia per gli alunni italiani che per gli alunni stranieri, un processo che ruota attorno al soggetto, al suo percorso e la sua storia di vita, alle relazioni instaurate nell'ambiente scolastico, all'atteggiamento e alle aspettative dei soggetti coinvolti nel processo educativo (alunno, genitori, insegnanti) ${ }^{14}$.

Per quanto riguarda i dati relativi al ritardo scolastico, gli alunni stranieri hanno da questo punto di vista percorsi decisamente più irregolari rispetto ai compagni italiani: per l'interpretazione di tale aspetto è basilare considerare il fatto che spesso gli alunni stranieri risultano in ritardo non perché il loro percorso scolastico annuale abbia avuto un esito negativo, ma perché le scuole attuano la scelta di inserire i nuovi iscritti, in particolare se neo-arrivati dall'estero, in una classe inferiore a quella corrispondente all'età anagrafica. Se tale prassi, che la normativa prevede a livello di possibilità con una precisa deliberazione del collegio docenti e tenendo conto di una serie di fattori, è sovente messa in atto per evitare agli studenti una bocciatura a fine anno, essa maschera tuttavia sovente un'impreparazione e una scarsa dotazione da parte delle scuole di strumenti adeguati per valutare le competenze degli alunni stranieri, percepiti più come portatori di uno svantaggio che va recuperato che come soggetti con un proprio bagaglio culturale, linguistico ed esperienziale.

Guardando ai dati relativi agli alunni provenienti da Ecuador e Perù rispetto al ritardo non legato ad una ripetenza ma ad una scelta della scuola, emerge come per entrambi le percentuali siano inferiori alla media per quanto riguarda l'inserimento in una classe corrispondente all'età anagrafica $(67,9$ per gli ecuadoriani e $68,5 \%$ per i peruviani, a fronte di una media regionale del $70,9 \%$ ) e, di contro, superiori alla media (che si attesta intorno al 29,1\%) per l'iscrizione ad una classe inferiore rispetto all'età $(32,1 \%$ per gli alunni ecuadoriani e $31,5 \%$ per quelli peruviani). Tale dato non sembra di facile interpretazione alla luce degli elementi a nostra disposizione: in alcuni casi appare legato alla percezione della vicinanza o lontananza culturale e linguistica dalla cultura italiana, come nel caso di altre cittadinanze, come quella cinese, in altri alla recente immigrazione sul territorio lombardo di alcune comunità, come quella ecuadoriana.

14 Tiana M. T., op cit., p. 73; Besozzi E., L'esperienza scolastica: mobilità, riuscita esignificati dell'istruzione, in Giovannini G., Queirolo-Palmas L., Una scuola in comune, Fondazione Agnelli, Torino 2002, pp. 64-65; Lillini R., Diversi ed eguali. Gli studenti di fronte al futuro, in Fravega R., Queirolo Palmas L. (a cura di), Classi meticce. Giovani, studenti, insegnanti nelle scuole delle migrazioni, Carocci, Roma 2003. 
Se ci soffermiamo sulle ripetenze, considerando la media regionale del $7,7 \%$ (percentuale che varia notevolmente in base agli ordini di scuola e che arriva al $21 \%$ nella secondaria di secondo grado) la situazione di alunni ecuadoriani e peruviani appare diversificata: se nell'anno scolastico 2003/2004 sono stati il $7,1 \%$ gli studenti ecuadoriani ad essere in ritardo per le ripetenze, lo sono stati ben il $12,4 \%$ degli alunni peruviani, la percentuale più alta fra le prime dieci cittadinanze. ${ }^{15}$ La spiegazione legata alla distanza culturale e linguistica non sembra essere soddisfacente, data la differente riuscita di ragazzi che appartengono ad una medesima area geografica; piuttosto, sembra incidere maggiormente sui risultati scolastici la nascita degli alunni in Italia o all'estero, e pertanto la precoce possibilità di contatto con la cultura italiana e l'acculturazione al suo sistema scolastico ${ }^{16}$. Infatti, prendendo in considerazione ancora una volta i dati relativi agli alunni di origine ecuadoriana e peruviana rispetto alla correlazione fra ripetenze e nascita in Italia o all'estero, è evidente come la percentuale dei ripetenti diminuisca notevolmente fra coloro che sono nati in Italia (Tab. 5).

Tab. 5. Nati in Italia e all'estero per cittadinanza e ripetenze: valori \%. Anno scol. 2003/2004

\begin{tabular}{l|c|c|}
\hline PAESI DI CITTADINANZA & RIPETENTI SUI NATI IN ITALIA \% & RIPETENTI SUI NATI ALL'ESTERO \% \\
\hline Marocco & 2 & 13 \\
Albania & 1 & 8 \\
Romania & 4 & 5 \\
Ecuador & 1 & 7 \\
Cina & 3 & 12 \\
Filippine & 2 & 8 \\
Peru' & 2 & 15 \\
India & 1 & 8 \\
Egitto & 4 & 9 \\
Pakistan & 3 & 12
\end{tabular}

Fonte: Besozzi E., Tiana M. T. (a cura di), Insieme a scuola 3, Fondazione Ismu, Milano 2005.

Anche per gli alunni con cittadinanza ecuadoriana e peruviana, così come per la popolazione scolastica straniera in generale, è possibile affermare che, a fronte di una frequenza regolare, il ritardo, che in gran parte è determinato dalle scelte delle scuole, è legato solo in parte a difficoltà nell'apprendimento e ad un profitto non soddisfacente; alla sua definizione contribuiscono in larga misura l'esperienza migratoria e la frammentazione e la discontinuità dei percorsi dei minori stranieri, i quali seguono la famiglia nelle scelte e nel progetto migratorio, che spesso prevede una forte mobilità. Inoltre, all'interno del contesto

15 I peruviani sono seguiti dagli alunni pakistani $(10,4 \%)$, cinesi $(9,1 \%)$ e marocchini $(8,9 \%)$.

16 Tiana M. T., op cit., p. 84-87. Tale aspetto non sembra invece incidere, come si vedrà in seguito, sulla scelta di proseguire gli studi. 
scolastico, il rendimento positivo o negativo degli alunni stranieri è indicatore dell'esistenza di approcci diversificati all'integrazione delle differenze e rimanda, a livello societario, ai rapporti fra immigrati e cultura dominante, al sistema di stratificazione sociale e al grado di apertura/chiusura rispetto all'uguaglianza delle opportunità di fronte all'istruzione ${ }^{17}$. Le difficoltà scolastiche vanno quindi analizzate facendo riferimento alla discontinuità culturale fra l'istituzione, la sua cultura e i soggetti, considerando che non si tratta solo di una differente livello di inculturazione e integrazione, ma di un rapporto di disparità fra gli alunni e l'istituzione in presenza della differenza culturale.

Come precedentemente accennato, uno dei dati maggiormente significativi che riguardano la popolazione scolastica proveniente dalle zone dell'America centro-meridionale riguarda la forte presenza di tali alunni all'interno della scuola secondaria di secondo grado, ordine di scuola in cui si evidenziano i nodi più problematici sia per quanto riguarda la scelta di proseguire gli studi da parte dei ragazzi stranieri e quindi l'assolvimento del diritto-dovere alla formazione, come stabilito dalla recente normativa italiana, sia per la caratterizzazione dei percorsi scolastici di questi ragazzi, spesso frammentati, sia per la scelta dell'indirizzo di studi, sia per la capacità degli istituti di attivarsi nel rispondere ai bisogni di tali studenti in termini di accoglienza, orientamento, integrazione.

Precedenti ricerche ${ }^{18}$ hanno messo in luce come l'orientamento degli alunni stranieri sia rivolto verso la prosecuzione degli studi, anche se in percentuali più contenute rispetto ai compagni italiani, con una grande consapevolezza riguardante gli sbocchi lavorativi possibili in base all'indirizzo di studi prescelto.

Se prendiamo in considerazione i dati di Ecuador e Perù, è possibile notare come il numero degli studenti iscritti all'istruzione superiore superi la numerosità degli alunni delle nazionalità il cui numero assoluto è più elevato sul totale degli ordini di scuola, come nel caso dell'Ecuador sulla Romania (603 alunni ecuadoriani contro i 582 rumeni) e del Perù sulle Filippine e la Cina (767 alunni peruviani contro i 481 cinesi e 385 filippini). Gli stessi peruviani negli istituti superiori sono in numero maggiore degli ecuadoriani, pur essendo meno numerosi se guardiamo alla totalità degli alunni. Incrociando tali dati con alcune variabili che ci possono permettere una prima interpretazione, quali il luogo di nascita dei minori - se in Italia o all'estero -, gli anni di permanenza in Italia per i nati all'estero e il grado di conoscenza della

17 Gobbo F., Pedagogia interculturale, Carocci, Roma 2004

18 Giovannini G., Queirolo-Palmas L. (a cura di), op. cit. 
lingua italiana, è possibile evidenziare come, in primo luogo, la quasi totalità degli alunni ecuadoriani e peruviani presenti negli istituti superiori della regione sia nata all'estero, con una percentuale di poco al di sopra della media regionale che è comunque del 93,8\%, attestando come la parte più consistente dei ragazzi con cittadinanza non italiana con più di quattordici anni abbia vissuto personalmente l'esperienza migratoria. L'essere nato all'estero è pertanto slegato dall' appartenenza ad una specifica cittadinanza, contrariamente invece a quanto emerge dai dati riguardanti i nati in Italia o all'estero degli alunni della scuola dell'infanzia e primaria, in cui vi sono evidenti scostamenti fra un paese di provenienza e l'altro, dovuti in gran parte alla forte presenza femminile di alcune comunità, alla stabilizzazione di nuclei familiari sul territorio, al ricorso ai ricongiungimenti familiari. Guardando, in specifico, all'anno di arrivo in Italia di coloro che sono nati all'estero, è possibile notare delle differenze fra chi proviene dall'Ecuador e chi dal Perù: se la maggior parte dei minori ecuadoriani sono giunti in Italia negli ultimi anni (il dati più significativi riguardano il 2002 e il 2003), confermando in qualche modo il fatto che si tratta di uno dei gruppi che si sono stabiliti sul territorio più recentemente ${ }^{19}$, per quanto riguarda i peruviani gli arrivi più consistenti si collocano negli anni fra il 1991 e il 1999. La stabilità sul territorio non sembra quindi essere direttamente correlata alla scelta di proseguire gli studi, essendo tale ipotesi disconfermata dai dati che riguardano gli alunni ecuadoriani.

Per quanto riguarda invece il livello di conoscenza della lingua italiana, lungo una scala che va da nessuna conoscenza alla conoscenza e uso di semplici termini, lettura e scrittura a livello elementare, sufficienti competenze, buonelottime competenze, gli alunni provenienti da Ecuador e Perù si collocano entrambi in particolare nella categoria sufficienti competenze; uno scostamento fra i due gruppi emerge invece per quanto riguarda una conoscenza della lingua buona/ottima, in cui prevalgono i peruviani (con il $18,5 \%$, dato superiore alla media regionale che è del $17,8 \%$ ), mentre nei livelli più bassi di conoscenza le percentuali maggiori si riferiscono agli ecuadoriani (la conoscenza buona/ottima si aggira solo intorno al 10,6\% mentre la percentuale maggiore è data da coloro che possiedono un livello elementare), segno che non sempre la vicinanza linguistica e culturale con il paese d'accoglienza è garanzia di un migliore e più veloce apprendimento della lingua italiana. Se prendiamo piuttosto in considerazione in particolare il livello di competenza linguistica di coloro che frequentano la scuola secondaria di secondo grado, a fronte di un dato regionale in cui raramente chi frequenta un istituto superiore non ha nessuna conoscenza linguistica,

19 Giovannini G., Le cittudinanze, in Besozzi E., Tiana M. T. (a cura di), op. cit., p. 65. 
il $42,8 \%$ degli ecuadoriani possiede sufficienti competenze, il $25,6 \%$ competenze elementari, il $16 \%$ competenze buone o ottime; tali dati evidenziano negli studenti dell'Ecuador una capacità linguistica migliore rispetto ai connazionali inseriti negli ordini di scuola inferiore. Lo stesso vale anche per gli studenti peruviani, fra i quali aumenta la percentuale di coloro che si collocano ad un livello buono/ottimo $(28,8 \%)$. Se la conoscenza linguistica non può certo essere considerata uno degli elementi determinanti nella scelta di proseguire gli studi, negli alunni provenienti da Ecuador e Perù essa sembra avere comunque un certo valore, tenendo anche in considerazione il fatto che gli istituti superiori sono ad oggi in genere meno attrezzati rispetto agli ordini di scuola inferiore per quanto riguarda l'alfabetizzazione e il sostegno scolastico degli studenti stranieri ${ }^{20}$. I fattori che giocano un ruolo nel definire e orientare i processi di scelta dei ragazzi stranieri rispetto alla scuola secondaria di secondo grado sono pertanto multidimensionali e sono legati, come mostrano altre indagini, oltre che alla conoscenza linguistica e al periodo di permanenza in Italia, al capitale sociale e culturale famigliare, al progetto migratorio, alle aspettative del ragazzo e della famiglia riguardo al futuro, che influiscono sull'investimento in istruzione, alla carriera scolastica pregressa ${ }^{21}$.

Le scelte scolastiche dei minori di Ecuador e Perù, rispetto alla tipologia di scuola frequentata, riffettono in generale sostanzialmente l'andamento regionale, con una presenza consistente all'interno degli istituti tecnici e professionali, maggiormente professionalizzanti e orientati all'inserimento nel mondo del lavoro, nonché spesso percepiti dagli stessi alunni come meno impegnativi per quanto riguarda lo studio. Le presenze di questi due gruppi di studenti nel sistema dei licei sono inferiori alle medie regionali, mentre si mostrano al di sopra della media per quanto riguarda istituti tecnici e professionali, anche se i peruviani sembrano prediligere i primi (in cui è iscritto il $51 \%$ degli studenti di questa cittadinanza che sono inseriti nella secondaria di secondo grado, contro il $42,3 \%$ degli alunni dell'Ecuador), mentre gli ecuadoriani nettamente i secondi (con il 50,2\% degli iscritti contro il $35,3 \%$ di chi proviene dal Perù).

20 Come evidenziato da E. Esparragoza (Esparragoza E., Guayaquil... Italia, in Fravega R., Queirolo Palmas L. (a cura di), op. cit.) per gli alunni ispanofoni ad una maggiore facilità di comprensione della lingua italiana corrisponde spesso una commistione fra la lingua madre e la lingua seconda, con il rischio che questa situazione si protragga sino alla perdita dello spagnolo e all'acquisizione di un italiano imperfetto. Su tali fattori giocano un ruolo la scolarizzazione pregressa dei minori e gli obiettivi del progetto migratorio dei genitori.

21 Giovannini G., Queirolo Palmas L. (a cura di), op. cit.; Lillini R, op. cit.; Bertozzi R., Dopo la terza media: le scelte, la riuscita e le aspettative dei giovani, in Giovannini G. (a cura di), La condizione dei minori stranieri in Italia, Fondazione Ismu, Milano 2005 
Tab. 6. Alunni ecuadoriani e peruviani nelle Scuole Secondarie di II grado della Lombardia per tipologia di istruzione. Anno scol. 2003/2004

\begin{tabular}{|c|c|c|c|c|c|c|c|c|c|}
\hline $\begin{array}{l}\text { PAESI DI } \\
\text { CITTADINANZA }\end{array}$ & $\begin{array}{l}\text { Licei } \\
\text { classici }\end{array}$ & $\begin{array}{c}\text { Licei } \\
\text { scientifici }\end{array}$ & $\begin{array}{c}\text { Licei } \\
\text { pedagogici }\end{array}$ & \begin{tabular}{|c|} 
Licei \\
linguistici
\end{tabular} & $\begin{array}{l}\text { Licei } \\
\text { artistici }\end{array}$ & $\begin{array}{l}\text { Istituti } \\
\text { d'arte. }\end{array}$ & $\begin{array}{l}\text { Istituti } \\
\text { tecnici }\end{array}$ & $\begin{array}{c}\text { Istituti } \\
\text { professionali }\end{array}$ & Totale \\
\hline Ecuador & 3 & 26 & 12 & - & 3 & 1 & 255 & 303 & 603 \\
\hline$\%$ & 0,5 & 4,3 & 2,0 & - & 0,5 & 0,2 & 42,3 & 50,2 & 100,0 \\
\hline Peru' & 6 & 63 & 19 & 6 & 7 & 4 & 391 & 271 & 767 \\
\hline$\%$ & 0,8 & 8,2 & 2,5 & 0,8 & 0,9 & 0,5 & 51,0 & 35,3 & 100,0 \\
\hline Totale & $\begin{array}{r}152 \\
17\end{array}$ & $\begin{array}{r}829 \\
95\end{array}$ & $\begin{array}{r}351 \\
40\end{array}$ & $\begin{array}{r}52 \\
0,6\end{array}$ & $\begin{array}{r}110 \\
1,3\end{array}$ & $\begin{array}{r}43 \\
0.5\end{array}$ & $\begin{array}{r}3.607 \\
41,3\end{array}$ & $\begin{array}{r}3.581 \\
41.0\end{array}$ & $\begin{array}{l}8.725 \\
100,0\end{array}$ \\
\hline
\end{tabular}

Fonte: Besozzi E., Tiana M. T. (a cura di), Insieme a scuola 3, Fondazione Ismu, Milano 2005.

Altro dato interessante riguarda la distribuzione delle presenze di tali alunni nelle scuole secondarie di secondo grado rispetto al territorio: contrariamente ad altre nazionalità, i ragazzi di Ecuador e Perù sono presenti in maggior numero nelle scuole delle città capoluogo in confronto a quelle dei comuni della provincia; questo verosimilmente per il fatto che entrambi questi gruppi sono maggiormente insediati nelle zone urbane.

Per quanto riguarda il genere, le femmine sono presenti in misura considerevole e proseguono gli studi molto più che per quanto riguarda altre cittadinanze: guardando infatti al dato che prende in esame le macro-aree di provenienza, fra gli alunni che provengono dall'America le femmine sono superiori ai maschi non solo nel sistema dei licei, ma anche negli istituti professionali. È un dato questo che riflette gli andamenti nazionali sia per quanto riguarda le ragazze straniere che italiane, entrambe le quali hanno anche percorsi scolastici più regolari e percentuali di successo maggiori rispetto ai compagni maschi.

\section{CONCLUSIONI}

I dati che danno conto dei percorsi e della riuscita scolastica dei giovani Latinos in Lombardia, e in particolare degli alunni ecuadoriani e peruviani, la cui numerosità è particolarmente significativa, mettono in luce alcune questioni alle quali la scuola italiana deve oggi rispondere per continuare ad essere efficace ambito di educazione e formazione delle giovani generazioni, rappresentate ora da soggetti le cui appartenenze culturali sono sempre più molteplici. Uno degli aspetti emergenti riguarda il fatto che il riferimento alla cittadinanza, così come la percezione della maggiore o minore vicinanza alla cultura italiana, oltre a non essere sufficiente a spiegare la complessità e la molteplicità dei fattori che definiscono i percorsi scolastici di questi alunni, può risultare in alcuni casi fuorviante, essendo spesso legata a stereotipi sociali o culturali strutturati attraverso l'individuazione di determinate caratteristiche 
derivate dalla cultura, utilizzate come categorie interpretative per spiegare le difficoltà scolastiche degli alunni stranieri ${ }^{22}$. La differenza di appartenenza, racchiusa entro i confini, sovente costruiti, della differenza culturale, diviene in tal modo sinonimo di mancanza; la scuola finisce quindi, interrogandosi sul perché gli studenti stranieri abbiano difficoltà nella riuscita, con non indagare sui motivi per cui essa stessa risulta poco efficace nei confronti di questi alunni. La sfida che la scuola deve oggi affrontare è quella di un superamento di stereotipi, pregiudizi, attese di basso profilo nei confronti degli alunni provenienti da culture diverse da quella italiana. Come precedentemente evidenziato, spesso l'istituzione scolastica avanza nei confronti degli studenti richieste di omologazione e concede alla differenza pochi spazi di espressione se non per i suoi aspetti più folclorici e rassicuranti, mettendo in luce la scarsa conoscenza dei contesti d'origine degli alunni da parte di insegnanti ed educatori e la scarsa capacità di riconoscere e valorizzare le competenze di partenza degli alunni stranieri. Tali considerazioni non valgono naturalmente allo stesso modo per tutti i contesti scolastici ${ }^{23}$, ma in generale si ravvisa l'esigenza di far fronte ad una serie di necessità, non rinviabili, per rispondere efficacemente ai bisogni diversificati di tutti gli alunni e prevenire possibili forme di disagio. Innanzitutto, è evidente come la scuola debba garantire pari opportunità, a partire da un'uguaglianza delle possibilità basata sulle peculiarità di ogni soggetto e sulla possibilità di una piena realizzazione del sé e delle proprie aspirazioni riguardo il futuro. In particolare, all'interno della scuola questo significa passare dalla garanzia dell'accesso alle strategie per il successo. È nell'orizzonte della garanzia del successo scolastico che, in secondo luogo, si deve porre un'attenzione particolare innanzitutto a creare interventi che permettano la diffusione delle "buone pratiche"24, che maggiormente si sono dimostrate efficaci nell'integrazione degli alunni stranieri e, inoltre, a garantire agli insegnanti e agli educatori una formazione specifica. In terzo luogo, appare necessario, attraverso la ricerca scientifica, analizzare sempre più approfonditamente i processi di inclusione ed esclusione scolastica e sociale, nonché ampliare la

22 Gobbo F., op. cit.

23 Molte scuole sono ormai uscite dalla gestione dell'emergenza e hanno inserito le attività interculturali all' interno dell'offerta formativa dei singoli istituti.

24 Si intende buona pratica di educazione interculturale un progetto o intervento che ha avuto la capacità di trasformare la realtà educativa e didattica, attivando una comunicazione fra culture. Nell'ambito della ricerca Ismu (si veda Besozzi E. (a cura di), L'educazione interculturale in Lombardia. Dal monitoraggio alle buone pratiche, Fondazione Ismu, Milano 2005) gli indicatori per individuare le buone pratiche sono stati: l'ampio coinvolgimento della realtà scolastica nel suo insieme, l'ampia rete di progetto e la circolazione dei prodotti e dei risultati, l'esplicitazione di obiettivi, metodi, risorse, la valutazione dei risultati, l'incremento di professionalità, l'innovazione di saperi, metodi, curricoli, il livello di documentazione e la riproducibilità dell'esperienza. 
conoscenza relativamente alle seconde generazioni e ai processi di scelta scolastica e lavorativa ${ }^{25}$.

$\mathrm{Si}$ ravvisa quindi la necessità di adottare un approccio multidimensionale alla riuscita scolastica, con il superamento, da parte della scuola e dei suoi insegnanti, di un'ottica di "assistenza" verso un'ottica di promozione alla piena realizzazione del sé di ogni alunno.

\section{BIBLIOGRAFIA}

BERTOZZI R., Dopo la terza media: le scelte, la riuscita e le aspettative dei giovani, in Giovannini G. (a cura di), La condizione dei minori stranieri in Italia, Fondazione Ismu, Milano 2005

BESOZZI E., Insegnare in una società multietnica: tra accoglienza, indifferenza e rifiuto, in Giovannini G. (a cura di), Allievi in classe stranieri in città. Una ricerca sugli insegnanti di scuola elementare di fronte all'immigrazione, F. Angeli, Milano 1996

BESOZZI E., L'esperienza scolastica: mobilità, riuscita e significati dell 'istruzione, in Giovannini G., Queirolo-Palmas L., Una scuola in comune, Fondazione Agnelli, Torino 2002

BESOZZI E., Percorsi ed esiti scolastici degli alunni con cittadinanza non italiana. Un'analisi dei fattori in gioco, in Ministero dell'Istruzione, dell'Università e della Ricerca, Indagine sugli esiti degli Alunni con Cittadinanza Non Italiana, gennaio 2005

BESOZZI E. (a cura di), L'educazione interculturale in Lombardia. Dal monitoraggio alle buone pratiche, Fondazione Ismu, Milano 2005

BESOZZI E., Tiana M. T. (a cura di), Insieme a scuola 3. La terza indagine regionale, Fondazione Ismu, Milano 2005

ESPARRAGOZA E., Guayaquil...Italia, in Fravega R., Queirolo Palmas L. (a cura di), Classi meticce. Giovani, studenti, insegnanti nelle scuole delle migrazioni, Carocci, Roma 2003

FRAVEGA R., Queirolo Palmas L. (a cura di), Classi meticce. Giovani, studenti, insegnanti nelle scuole delle migrazioni, Carocci, Roma 2003

GIOVANNINI G., Queirolo-Palmas L., Una scuola in comune, Fondazione Agnelli, Torino 2002

GIOVANNINI G., Le cittadinanze, in Besozzi E., Tiana M. T. (a cura di), Insieme a scuola 3. La terza indagine regionale, Fondazione Ismu, Milano 2005

GIOVANNINI G. (a cura di), La condizione dei minori stranieri in Italia, Fondazione Ismu, Milano 2005

GOBBO F., Pedagogia interculturale, Carocci, Roma 2004

LILLINI R., Diversi ed eguali. Gli studenti di fronte al futuro, in Fravega R., Queirolo Palmas L. (a cura di), Classi meticce. Giovani, studenti, insegnanti nelle scuole delle migrazioni, Carocci, Roma 2003

MAZZİ D., Presentazione dei risultati generali, in Besozzi E., Tiana M. T. (a cura di), Insieme a scuola 3. La terza indagine regionale, Fondazione Ismu, Milano 2005

TIANA M.T., I percorsi e la riuscita scolastica, in Besozzi E., Tiana M. T. (a cura di), Insieme a scuola 3. La terza indagine regionale, Fondazione Ismu, Milano 2005 